\title{
Effect of Statin Use on Acute Kidney Injury Risk Following Coronary Artery Bypass Grafting
}

\author{
J. Bradley Layton, PhD ${ }^{a, b}$, Abhijit V. Kshirsagar, MD, MPH ${ }^{b}$, Ross J. Simpson Jr., MD, PhDc, \\ Virginia Pate, MS ${ }^{a}$, Michele Jonsson Funk, PhD ${ }^{a}$, Til Sturmer, MD, MPH, PhD ${ }^{a}$, and M. Alan \\ Brookhart, PhD $^{\mathrm{a}}$ \\ aDepartment of Epidemiology, University of North Carolina at Chapel Hill, Chapel Hill, NC \\ bDivision of Nephrology, University of North Carolina at Chapel Hill, Chapel Hill, NC \\ 'Division of Cardiology, Department of Medicine, University of North Carolina at Chapel Hill, \\ Chapel Hill, NC
}

\begin{abstract}
Acute kidney injury (AKI) is a serious complication of cardiovascular surgery. While some nonexperimental studies suggest statin use may reduce post-surgical AKI, methodological differences in study designs leave uncertainty regarding the reality or magnitude of the effect. We estimated the effect of pre-operative statin initiation on post-coronary artery bypass graft (CABG) AKI using an epidemiologic approach more closely simulating a randomized controlled trial in a large CABG patient population. We utilized healthcare claims from large, employer-based and Medicare insurance databases for the years 2000 - 2010. To minimize healthy user bias, we identified patients undergoing non-emergency CABG who either newly initiated a statin within 20 days prior to surgery or were unexposed for 200+ days prior to CABG. AKI was identified within 15 days following CABG. We calculated multivariable adjusted risk ratios (RR) and 95\% confidence intervals (CI) with Poisson regression. Analyses were repeated using propensity score methods adjusted for clinical and healthcare utilization variables. We identified 17,077 CABG patients. Post-CABG AKI developed in $3.4 \%$ of statin initiators and $6.2 \%$ of non-initiators. After adjustment, we observed a protective effect of statin initiation on AKI $(\mathrm{RR}=0.78,95 \%$ CI 0.63 , $0.96)$. This effect differed by age: $\succ 65$ years, $R R=0.91$ (95\% CI: $0.68,1.20) ;<65$ years, $R R=0.62$ (95\% CI: $0.45,0.86$ ), although AKI was more common in the older age group ( 7.7 vs. $4.0 \%$ ). In conclusion, statin initiation immediately prior to CABG may modestly reduce the risk of postoperative AKI, particularly in younger $\mathrm{CABG}$ patients.
\end{abstract}

\section{Keywords}

statins; bypass; heart surgery; acute kidney injury; pharmacoepidemiology

\footnotetext{
C 2012 Excerpta Medica, Inc. All rights reserved.

Contact info: Bradley Layton, 725 Martin Luther King Jr. Blvd., Office 234, Campus Box 7590, Chapel Hill, NC 27599-7590, Phone: 919-843-7151, Fax: 919-966-4251, blayton@unc.edu.

Publisher's Disclaimer: This is a PDF file of an unedited manuscript that has been accepted for publication. As a service to our customers we are providing this early version of the manuscript. The manuscript will undergo copyediting, typesetting, and review of the resulting proof before it is published in its final citable form. Please note that during the production process errors may be discovered which could affect the content, and all legal disclaimers that apply to the journal pertain.
} 


\section{Introduction}

Statins may have anti-inflammatory ${ }^{1}$ and endothelial stabilizing ${ }^{2}$ pleiotropic effects with potential benefits on kidney function ${ }^{3}$, motivating investigation for protective effects against post-surgical kidney injury ${ }^{3-15}$. However, studies have employed widely varying definitions of pre-operative statin use, including: prescribed statin use at the time of surgery ${ }^{4-6,8}$; administration the day of or the day before surgery ${ }^{11,12,15}$; or pharmacy dispensing within 90 days prior to $\mathrm{CABG}^{14}$. Many of these definitions fail to consider history or duration of statin use, which may introduce bias due to the healthy user effect ${ }^{16}$ whereby differences in healthy behaviors between long-term medication users and non-users may lead to exaggerated estimates of the benefits of preventive medications. These important methodological concerns limit the ability to distinguish whether observed results are direct beneficial effect of statins, or result from unmeasured behavioral differences in long-term users of statins. Yet, acute kidney injury (AKI) remains a serious complication of coronary bypass grafting $(\mathrm{CABG})$, resulting in both short- and long-term consequences, including chronic kidney disease (CKD), end-stage renal disease, or death ${ }^{17}$. Understanding the effect of pre-operative statin use could allow clinicians to modify the risk of an outcome for which there are currently no proven interventions. In a cohort of patients undergoing planned CABG surgery, we compared post-surgery AKI risk among patients initiating a statin immediately prior to surgery to patients not initiating statins using a modern epidemiologic study design and analysis aimed at minimizing confounding bias.

\section{Methods}

Individuals undergoing CABG surgery between the years 2000 to 2010 were identified in Thomson Reuters' MarketScan ${ }^{\circledR}$ Commercial Claims and Encounters and Medicare Supplemental and Coordination of Benefits databases (Thomson Reuters (Healthcare) Inc., 2011). These databases are a compilation of insurance billing data for employees, dependents and retirees from across the United States with employer-based primary or Medicare supplemental insurance coverage (ages $\geq 65$ years). Adjudicated, paid inpatient, outpatient, and pharmacy claims, as well as enrollment information, are included in the databases. This study was exempted from further review by the University of North Carolina Institutional Review Board.

All patients $\geq 18$ years with inpatient procedure claims for CABG, having 200 days of continuous plan enrollment prior to hospital admission for $\mathrm{CABG}$, were identified. If an individual had multiple eligible CABG surgeries, only the first was considered. The 20 days immediately prior to the date of hospital admission were considered the exposure window, during which statin initiation was assessed. The 180 days prior to the exposure window were considered the washout period, during which the absence of any statin prescription was required (see Figure 1). We required at least 1 pharmacy claim for any non-statin medication during the washout period to ensure pharmacy benefit utilization.

Patients with inpatient or outpatient diagnosis codes for AKI, unspecified renal failure, or end-stage renal disease in the 200 days before CABG surgery were excluded. To restrict to planned CABG procedures by removing individuals with emergency surgeries, we excluded patients with: inpatient claims for MI or unstable angina during the 20-day exposure period; $\mathrm{CABG}$ occurring after the fifth day of hospitalization; or an angiogram in the 3 days prior to CABG surgery.

Statin initiation was defined as having a pharmacy dispensing claim for any statin during the exposure window without any statin claims during the preceding baseline period. Non-users had no observable statin use during the exposure or baseline windows and were required to 
have an outpatient physician's office visit during the exposure window to ensure healthcare utilization.

Baseline covariates for multivariable regression and propensity score models included claims for diagnoses, procedures, prevalent medication use, and pre-operative initiation of other, non-statin medications. Included covariates are shown in Table 1. Baseline diagnoses and procedures were assessed in the 200 days prior to hospital admission for CABG and during the hospitalization up to the day of surgery, and included: age; sex; year of surgery; number of grafts in surgery; diagnoses of cardiovascular conditions; indicators of CVD management; acute cardiovascular events and procedures; evidence of renal conditions; number of emergency department visits; and number of hospitalizations. Claims for medications during the washout period were considered prevalent medication use. If the medications were newly initiated during the exposure window without use during the washout period, the medications were considered newly-initiated and considered separately in the analysis.

Inpatient claims in the 15 days post-CABG were searched for ICD-9-CM diagnosis codes for AKI (584.5 - 584.9). Sensitivity analyses were performed employing a broader definition of kidney failure (any of the following diagnosis codes: acute renal failure, 584.5 -584.9; end stage renal disease, 585.6; or unspecified renal failure, 586).

We estimated the association between statin initiation and AKI using multivariable Poisson regression, resulting in adjusted risk ratios (RR) and $95 \%$ confidence intervals $(\mathrm{CI})^{18}$. We also performed regression analyses employing stabilized inverse probability of treatment weighting (IPTW) ${ }^{19}$. Multivariable logistic regression was used to estimate the predicted probability of initiating a statin, or propensity score (PS), for each individual in the sample using the pre-specified covariates described above. To exclude patients treated contrary to prediction as their extreme weights may disproportionately influence the effect measure estimate ${ }^{20}$, we trimmed individuals with a PS less than the $1^{\text {st }}$ percentile of the treated, or greater than the $99^{\text {th }}$ percentile of the untreated. The PS was then used to calculate the IPTW in the remaining patients, and the weights were applied to a Poisson regression model.

Lastly, we performed 1-to-1 PS matching using a greedy matching algorithm ${ }^{21}$ where nonusers were matched to statin initiators by PS to the fifth decimal place, if possible. We then estimated RR using regression models in the remaining matched individuals.

Models were run separately in the 2 databases: commercial insurance (ages 40-64 years) and Medicare supplementary insurance (ages $\Varangle 65$ years). We also repeated the analyses in pre-specified subgroups - by gender, in those without CKD, and older age. Analyses were performed using SAS 9.2 (SAS Institute Inc., Cary, NC).

Sensitivity analyses were performed by varying the length of the exposure window before CABG (10 to 30 days) to observe if the effect may be dependent on the length of time on statin prior to CABG. To estimate the extent to which medication initiation is simply a proxy for better pre-surgical care, the entire analysis was repeated considering beta-blocker initiation as a negative control exposure ${ }^{22}$ rather than statin initiation. Beta-blockers are preventive cardiovascular medication with a similar behavioral profile and user population as statins, but they are not thought to confer a protective effect against post-operative AKI. Therefore, if a protective effect was observed among the beta-blocker initiators, it can be assumed that our study design did not adequately address the healthy user effect and other sources of confounding bias. 


\section{Results}

We identified 149,696 individuals with CABG surgeries with 200 days of baseline enrollment. After applying exclusion criteria, our sample consisted of 17,077 patients: 3,085 of whom newly initiated a statin within 20 days prior to CABG admission, and 13,992 who were free of any statin use. For the distribution of day of statin initiation prior to CABG among the initiators, see Figure 2. Distributions of demographic and clinical variables by statin status are shown in Table 1. The sample was predominantly male. Statin initiators were predominantly commercially-insured, whereas statin non-initiators were as likely to be covered by Medicare or commercial insurance. Variables were generally well-balanced across treatment groups, with the notable exceptions that initiators: received more cardiac stress tests or angiograms; had more diagnoses of hyperlipidemia and ischemic heart disease; and to co-initiated more other cardiovascular medications. While these characteristics may be associated with increased CVD risk, in claims data all of these factors may also be seen as markers of better disease management, preventive healthcare utilization, or pre-operative care.

Post-CABG AKI codes occurred in $871(6.2 \%)$ non-initiators and 104 (3.4\%) initiators. Unadjusted regression models yielded a highly-protective effect measure estimate, but adjustment attenuated the estimate to $\mathrm{RR}=0.78$ (95\% CI: $0.63,0.96)$ (see Table 2). When the analysis was repeated with trimmed IPTW weighted models and PS matching, the results were very similar (see Table 2).

However, when stratifying the effect measure estimates by age, the relative protective effect was more pronounced in individuals aged $<65$ years than older individuals (see Table 3 ). AKI was much more common in the older age group (7.7\%) than the younger age group $(4.0 \%)$. No differences were seen in other subgroups.

When the length of exposure window before CABG was varied, the effect measure estimate remained constant. Additionally, when a wider definition of kidney failure was employed, the results remained essentially unchanged (see Table 2).

When the analysis was repeated considering beta-blocker initiation in the place of statin initiation, we observed no protective effect of beta blockers initiation against post-CABG AKI, with multivariable adjusted models yielding RR $=0.96$ (95\% CI: $0.84,1.10)$ and IPTW models yielding RR=1.02 (95\% CI: 0.87, 1.19).

\section{Discussion}

In this cohort study of over 17,000 patients, we found that initiation of a statin prior to nonemergent CABG surgery was associated with a reduced risk of post-surgical AKI. The results were robust throughout sensitivity analyses and estimation methods.

We observed effect measure modification by age. The protective effect was much more pronounced in younger individuals. However, those under 65 years experienced many fewer post-CABG AKI events overall, suggesting that the absolute benefit of statin treatment may not be as large as the RR suggests ${ }^{23}$.

Statins lower low-density lipoprotein cholesterol, reducing the risk of CVD. Randomized trials have also demonstrated that peri-operative statin use is associated with decreased myocardial ischemia ${ }^{24}$ and atrial fibrillation ${ }^{25}$, which could indirectly benefit kidney function by maintaining renal perfusion during and immediately following surgery. However, the elderly are at higher AKI risk for a variety of reasons ${ }^{26}$, and statins' benefit may be diluted in such an at-risk population. 
Other estimates of the effect of peri-operative statin use on post-operative AKI have ranged from strongly protective ${ }^{8}$ to null ${ }^{4,10,13}$. Our estimate is comparable to other studies of the subject $^{3,14}$, but differs from the null result of a recent, small randomized trial ${ }^{10}$ restricted to high-AKI risk patients. We saw little protective effect in older patients, but observed the protective effect primarily in the younger, low-risk patients, which could explain the difference in results.

Non-randomized studies of preventive medications are potentially subject to bias which can inflate protective effect measure estimates. Yet, through the implementation of a rigorous methodology, our study helps to more accurately quantify the potential benefit of statins in 4 ways. First, we adopted a design explicitly aimed at minimizing the healthy user effect, a potentially important bias in non-experimental statin studies. We restricted our study to new users who initiated a statin immediately prior to surgery and compared these with patients not initiating a statin, removing bias created by comparing long-term, adherent users to nonusers.

Second, we excluded emergency CABG surgeries, resulting in a relatively homogeneous patient population of those with planned CABG surgeries. We speculated that statin initiation in these patients would be more dependent on physician preference or protocol than clinical factors or patient characteristics.

Third, we adjusted for a wide array of clinical characteristics, including complexity of surgery. Prevalent medication use and concurrent medication initiation were considered as separate covariates. Prevalent medication use was very similar between the 2 treatment groups, but statin initiators tended to co-initiate other medications more frequently. While statin initiation immediately prior to surgery may be indicative of more aggressive disease management or access to care, considering other peri-operative medication initiation allowed us to more accurately assess pre-operative care and adjust for differences which may exist between the treatment groups.

Lastly, we examined the effect of beta-blocker initiation on post-CABG AKI as a negative control. Beta blocker initiation was not associated with a reduced AKI risk, suggesting the observed effect estimate among the statin users may be a real effect, not simply unmeasured behavioral or clinical factors for which any medication initiation serves as a proxy ${ }^{27}$.

Nevertheless, these study findings should be interpreted in the context of the following limitations. First, despite our attempts to create comparable treatment groups, we observed differences-e.g. more healthcare utilization, CVD management, and concurrent medication initiation - among the statin users which may indicate better health status, or healthcare quality and access, and thus potentially better outcomes among statin users. Although we controlled for these observed factors in our analysis, the potential for unmeasured confounding remains. For example, measures of baseline glomerular function are unavailable in claims; diagnosis codes for CKD and number of procedure claims for measures of glomerular function were used as proxies, but they may not fully capture baseline renal impairment, which is a strong risk factor for $\mathrm{AKI}^{28}$. However, while glomerular function is a strong predictor of AKI, it is not obviously a predictor of statin initiation, and therefore may not be a strong confounder of the association.

Secondly, some of the included variables have been shown to have poor validity, such as $\mathrm{CKD}^{29}$ which would allow for some residual confounding by baseline renal impairment to remain. Similarly, the sensitivity AKI billing codes has been shown to be very poor, yet the specificity is very high ${ }^{29}$. While the insensitive AKI definition may underestimate true AKI occurrence, under the assumption of nondifferential misclassification of the outcome 
between the treatment groups, relative effect measures, such as the RRs calculated in this study, should be unbiased ${ }^{30}$.

Third, these results may not be generalizable to all CABG patients. The majority of patients undergoing $\mathrm{CABG}$ were prevalent statin users, and many others had urgent or emergency surgeries, thus not meeting our inclusion criteria. However, emergency CABG patients likely do not have time to initiate a statin prior to surgery. The resulting patients are those of most interest: it is among statin naive patients that statin intervention is possible and the potential benefits of initiation need to be determined.

As researchers continue to investigate and suggest additional protective and beneficial statin effects, careful consideration must be given to the designs of non-experimental studies to ensure they are not subject to common biases in studies of preventive medications. After considering the timing of statin initiation, observing the entire length of statin treatment, measuring important markers of pre-clinical care such as medication initiation, and matching non-users to users on healthcare utilization, our study supports the hypothesis that prescribing a statin prior to $\mathrm{CABG}$ in those not already receiving statin therapy may modestly attenuate the incidence of post-CABG AKI, particularly among younger patients. While many $C A B G$ patients will already be receiving statin therapy, those not yet receiving it may benefit from initiation prior to surgery.

\section{Acknowledgments}

Funding: This project was supported by an NIH training grant in renal epidemiology (5 T32 DK007750-13) to Dr. Layton administered through the UNC Kidney Center

\section{References}

1. Chello M, Patti G, Candura D, Mastrobuoni S, Di Sciascio G, Agro F, Carassiti M, Covino E. Effects of atorvastatin on systemic inflammatory response after coronary bypass surgery. Crit Care Med. 2006; 34:660-667. [PubMed: 16505650]

2. Giusti-Paiva A, Martinez MR, Felix JV, da Rocha MJ, Carnio EC, Elias LL, Antunes-Rodrigues J. Simvastatin decreases nitric oxide overproduction and reverts the impaired vascular responsiveness induced by endotoxic shock in rats. Shock. 2004; 21:271-275. [PubMed: 14770041]

3. Mithani S, Kuskowski M, Slinin Y, Ishani A, McFalls E, Adabag S. Dose-dependent effect of statins on the incidence of acute kidney injury after cardiac surgery. Ann Thorac Surg. 2011; 91:520-525. [PubMed: 21256305]

4. Argalious M, Xu M, Sun Z, Smedira N, Koch CG. Preoperative statin therapy is not associated with a reduced incidence of postoperative acute kidney injury after cardiac surgery. Anesth Analg. 2010; 111:324-330. [PubMed: 20375302]

5. Pan W, Pintar T, Anton J, Lee VV, Vaughn WK, Collard CD. Statins are associated with a reduced incidence of perioperative mortality after coronary artery bypass graft surgery. Circulation. 2004; 110:II45-49. [PubMed: 15364837]

6. Clark LL, Ikonomidis JS, Crawford FA Jr, Crumbley A 3rd, Kratz JM, Stroud MR, Woolson RF, Bruce JJ, Nicholas JS, Lackland DT, Zile MR, Spinale FG. Preoperative statin treatment is associated with reduced postoperative mortality and morbidity in patients undergoing cardiac surgery: an 8-year retrospective cohort study. J Thorac Cardiovasc Surg. 2006; 131:679-685. [PubMed: 16515923]

7. Le Manach Y, Ibanez Esteves C, Bertrand M, Goarin JP, Fleron MH, Coriat P, Koskas F, Riou B, Landais P. Impact of preoperative statin therapy on adverse postoperative outcomes in patients undergoing vascular surgery. Anesthesiology. 2011; 114:98-104. [PubMed: 21178671]

8. Virani SS, Nambi V, Polsani VR, Lee VV, Elayda M, Kohsaka S, Pan W, Reul RM, Wilson JM, Petersen LA, Willerson JT, Ballantyne CM. Preoperative statin therapy decreases risk of postoperative renal insufficiency. Cardiovasc Ther. 2010; 28:80-86. [PubMed: 20398096] 
9. Welten GM, Chonchol M, Schouten O, Hoeks S, Bax JJ, van Domburg RT, van Sambeek M, Poldermans D. Statin use is associated with early recovery of kidney injury after vascular surgery and improved long-term outcome. Nephrol Dial Transplant. 2008; 23:3867-3873. [PubMed: 18628367]

10. Prowle JR, Calzavacca P, Licari E, Ligabo EV, Echeverri JE, Haase M, Haase-Fielitz A, Bagshaw SM, Devarajan P, Bellomo R. Pilot double-blind, randomized controlled trial of short-term atorvastatin for prevention of acute kidney injury after cardiac surgery. Nephrology (Carlton). 2012; 17:215-224. [PubMed: 22117606]

11. Huffmyer JL, Mauermann WJ, Thiele RH, Ma JZ, Nemergut EC. Preoperative statin administration is associated with lower mortality and decreased need for postoperative hemodialysis in patients undergoing coronary artery bypass graft surgery. J Cardiothorac Vasc Anesth. 2009; 23:468-473. [PubMed: 19157909]

12. Tabata M, Khalpey Z, Pirundini PA, Byrne ML, Cohn LH, Rawn JD. Renoprotective effect of preoperative statins in coronary artery bypass grafting. Am J Cardiol. 2007; 100:442-444. [PubMed: 17659925]

13. Kor DJ, Brown MJ, Iscimen R, Brown DR, Whalen FX, Roy TK, Keegan MT. Perioperative statin therapy and renal outcomes after major vascular surgery: a propensity-based analysis. $\mathrm{J}$ Cardiothorac Vasc Anesth. 2008; 22:210-216. [PubMed: 18375322]

14. Molnar AO, Coca SG, Devereaux PJ, Jain AK, Kitchlu A, Luo J, Parikh CR, Paterson JM, Siddiqui N, Wald R, Walsh M, Garg AX. Statin use associates with a lower incidence of acute kidney injury after major elective surgery. J Am Soc Nephrol. 2011; 22:939-946. [PubMed: 21493769]

15. Brunelli SM, Waikar SS, Bateman BT, Chang TI, Lii J, Garg AX, Winkelmayer WC, Choudhry NK. Preoperative Statin Use and Postoperative Acute Kidney Injury. Am J Med. 2012

16. Brookhart MA, Patrick AR, Dormuth C, Avorn J, Shrank W, Cadarette SM, Solomon DH. Adherence to lipid-lowering therapy and the use of preventive health services: an investigation of the healthy user effect. Am J Epidemiol. 2007; 166:348-354. [PubMed: 17504779]

17. Ishani A, Nelson D, Clothier B, Schult T, Nugent S, Greer N, Slinin Y, Ensrud KE. The magnitude of acute serum creatinine increase after cardiac surgery and the risk of chronic kidney disease, progression of kidney disease, and death. Arch Intern Med. 2011; 171:226-233. [PubMed: 21325112]

18. Zou G. A modified poisson regression approach to prospective studies with binary data. Am J Epidemiol. 2004; 159:702-706. [PubMed: 15033648]

19. Cole SR, Hernan MA. Constructing inverse probability weights for marginal structural models. Am J Epidemiol. 2008; 168:656-664. [PubMed: 18682488]

20. Sturmer T, Rothman KJ, Avorn J, Glynn RJ. Treatment effects in the presence of unmeasured confounding: dealing with observations in the tails of the propensity score distribution--a simulation study. Am J Epidemiol. 2010; 172:843-854. [PubMed: 20716704]

21. Parsons, LS. Reducing bias in a propensity score matched-pair sample using greedy matching techniques. SAS Institute Inc; 2001. p. 214-226.

22. Brookhart MA, Patrick AR, Shrank WH, Dormuth CR. Validating studies of adherence through the use of control outcomes and exposures. Am J Hypertens. 2010; 23:110. [PubMed: 20087324]

23. Rothman KJ, Poole C. A strengthening programme for weak associations. Int J Epidemiol. 1988; 17:955-959. [PubMed: 3225112]

24. Schouten O, Boersma E, Hoeks SE, Benner R, van Urk H, van Sambeek MR, Verhagen HJ, Khan NA, Dunkelgrun M, Bax JJ, Poldermans D. Fluvastatin and perioperative events in patients undergoing vascular surgery. N Engl J Med. 2009; 361:980-989. [PubMed: 19726772]

25. Patti G, Chello M, Candura D, Pasceri V, D’Ambrosio A, Covino E, Di Sciascio G. Randomized trial of atorvastatin for reduction of postoperative atrial fibrillation in patients undergoing cardiac surgery: results of the ARMYDA-3 (Atorvastatin for Reduction of MYocardial Dysrhythmia After cardiac surgery) study. Circulation. 2006; 114:1455-1461. [PubMed: 17000910]

26. Rao, MK.; Anderson, S. The Kidney in Aging. In: Greenberg, A., editor. Primer on Kidney Diseases. Philadelphia: Saunders Elsevier; 2009. p. 413-419. 
27. Patrick AR, Shrank WH, Glynn RJ, Solomon DH, Dormuth CR, Avorn J, Cadarette SM, Mogun $\mathrm{H}$, Brookhart MA. The association between statin use and outcomes potentially attributable to an unhealthy lifestyle in older adults. Value Health. 2011; 14:513-520. [PubMed: 21669377]

28. Charytan DM, Yang SS, McGurk S, Rawn J. Long and short-term outcomes following coronary artery bypass grafting in patients with and without chronic kidney disease. Nephrol Dial Transplant. 2010; 25:3654-3663. [PubMed: 20551089]

29. Vlasschaert ME, Bejaimal SA, Hackam DG, Quinn R, Cuerden MS, Oliver MJ, Iansavichus A, Sultan N, Mills A, Garg AX. Validity of administrative database coding for kidney disease: a systematic review. Am J Kidney Dis. 2011; 57:29-43. [PubMed: 21184918]

30. Greenland, S.; Lash, TL. Bias analysis. In: Rothman, KJ.; Greenland, S.; Lash, TL., editors. Modern Epidemiology. Philadelphia, PA: Lippincott Williams \& Wilkins; 2008. 


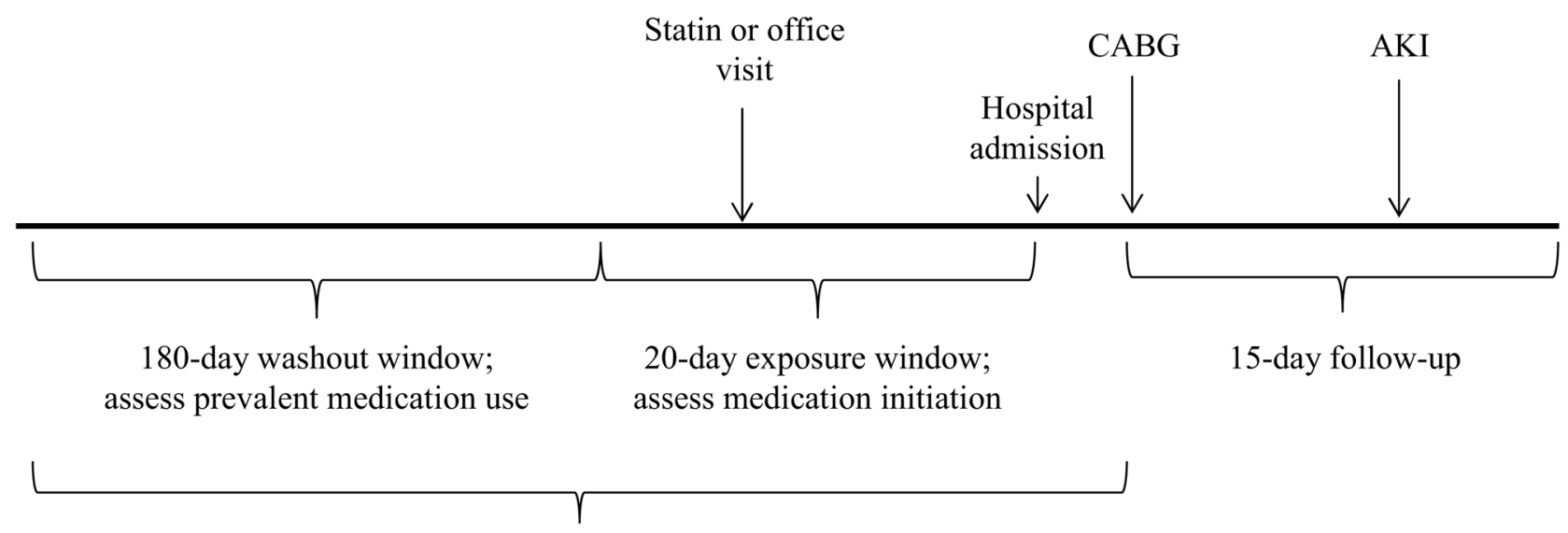

Assess diagnoses and procedures

Figure 1.

Cohort schematics for pre-coronary artery bypass graft statin initiation 


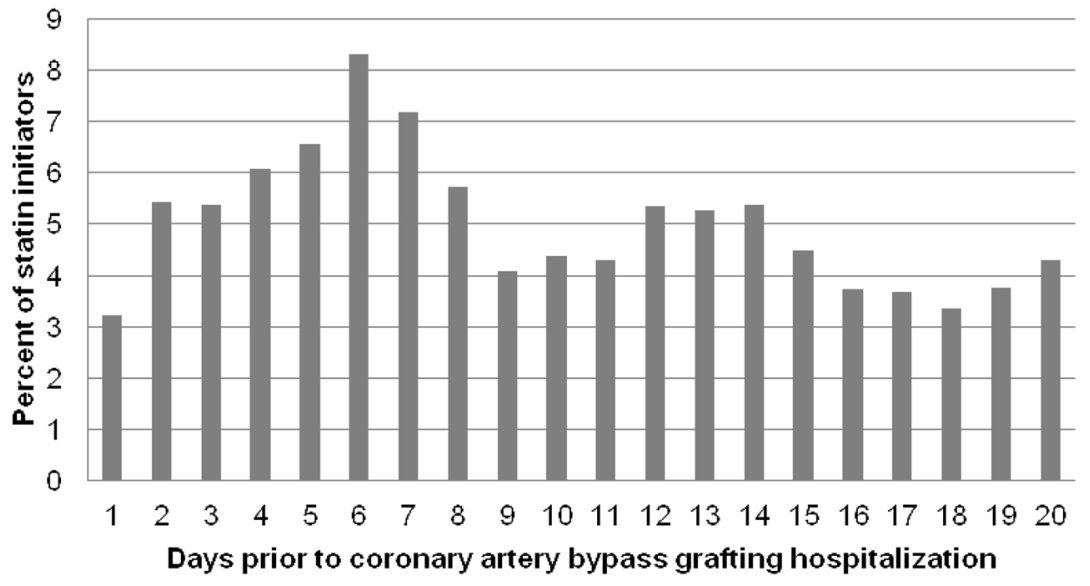

Figure 2.

Distribution of statin initiation by day prior to $\mathrm{CABG}$ surgery 
Table 1

Baseline demographics and clinical characteristics by statin initiation status

\begin{tabular}{|c|c|c|}
\hline Variable & Statin non-initiator $(n=13,992)$ & Statin initiator $(\mathbf{n}=\mathbf{3 , 0 8 5})$ \\
\hline Male & $10,394(74.3 \%)$ & $2,453(79.5 \%)$ \\
\hline Mean age, standard deviation (SD) & $65.4(11.0)$ & $(62.5)(10.0)$ \\
\hline \multicolumn{3}{|l|}{ MarketScan Database } \\
\hline Commercial Claims and Encounters Database & $7,135(51.0 \%)$ & $1,978(64.1 \%)$ \\
\hline Medicare Supplementary Database & $6,857(49.0 \%)$ & $1,107(35.9 \%)$ \\
\hline \multicolumn{3}{|l|}{ HEALTHCARE UTILIZATION } \\
\hline Mean day of hospitalization on which CABG is performed (SD) & $0.88(1.26)$ & $0.49(0.99)$ \\
\hline Mean number of lipid tests $(\mathrm{SD})^{*}$ & $0.68(1.11)$ & $0.84(1.12)$ \\
\hline Mean number of creatinine measurements (SD) ${ }^{*}$ & $0.04(0.37)$ & $0.03(0.25)$ \\
\hline Mean number of hospitalizations (SD) ${ }^{*}$ & $0.67(0.76)$ & $0.47(0.70)$ \\
\hline Mean number of emergency department visits $(\mathrm{SD})^{*}$ & $0.14(0.49)$ & $0.11(0.43)$ \\
\hline \multicolumn{3}{|l|}{ CARDIOVASCULAR DISEASE MANAGEMENT } \\
\hline Angiography performed & $10,492(75.0 \%)$ & $2,634(85.4 \%)$ \\
\hline Cardiac stress test performed & $8,381(59.9 \%)$ & $2,166(70.2 \%)$ \\
\hline Echocardiograph & $8,030(57.4 \%)$ & $1,723(55.9 \%)$ \\
\hline \multicolumn{3}{|l|}{ CARDIOVASCULAR \& COMORBID CONDITIONS } \\
\hline \multicolumn{3}{|l|}{ Number of vessels bypassed during surgery } \\
\hline $1-2$ & $5,265(37.6 \%)$ & $951(30.8 \%)$ \\
\hline $3-5$ & $7,565(54.1 \%)$ & $1,779(57.7 \%)$ \\
\hline $6+$ & $862(6.2 \%)$ & $259(8.4 \%)$ \\
\hline Diabetes mellitus & $4,170(29.8 \%)$ & $813(26.4 \%)$ \\
\hline Chronic kidney disease & $109(0.8 \%)$ & $13(0.4 \%)$ \\
\hline \multicolumn{3}{|l|}{ Other kidney disease } \\
\hline Proteinuria & $70(0.5 \%)$ & $13(0.4 \%)$ \\
\hline Hypertension & $7,208(51.5 \%)$ & $1,596(51.7 \%)$ \\
\hline Hyperlipidemia & $4,550(32.5 \%)$ & $1,283(41.6 \%)$ \\
\hline Other ischemic heart disease & $12,811(91.6 \%)$ & $2,972(96.3 \%)$ \\
\hline Atrial fibrillation & $1,422(10.2 \%)$ & $163(5.3 \%)$ \\
\hline \multicolumn{3}{|l|}{ ACUTE EVENTS IN PREVIOUS 6 MONTHS } \\
\hline Recent myocardial infarction ${ }^{\dagger}$ & $484(3.5 \%)$ & $108(3.5 \%)$ \\
\hline History of myocardial infarction & $364(2.6 \%)$ & $83(2.7 \%)$ \\
\hline Unstable angina ${ }^{\dagger}$ & $1,743(12.5 \%)$ & $464(15.0 \%)$ \\
\hline Stroke & $3,723(26.6 \%)$ & $746(24.2 \%)$ \\
\hline Insertion of a coronary stent & $236(1.7 \%)$ & $51(1.7 \%)$ \\
\hline Angioplasty & $228(1.6 \%)$ & $50(1.6 \%)$ \\
\hline \multicolumn{3}{|l|}{ PREVALENT MEDICATION USE DURING BASELINE } \\
\hline Angiotensin converting enzyme inhibitors & $4,349(31.1 \%)$ & $916(29.7 \%)$ \\
\hline Angiotensin receptor blockers & $2,212(15.8 \%)$ & $434(14.1 \%)$ \\
\hline
\end{tabular}




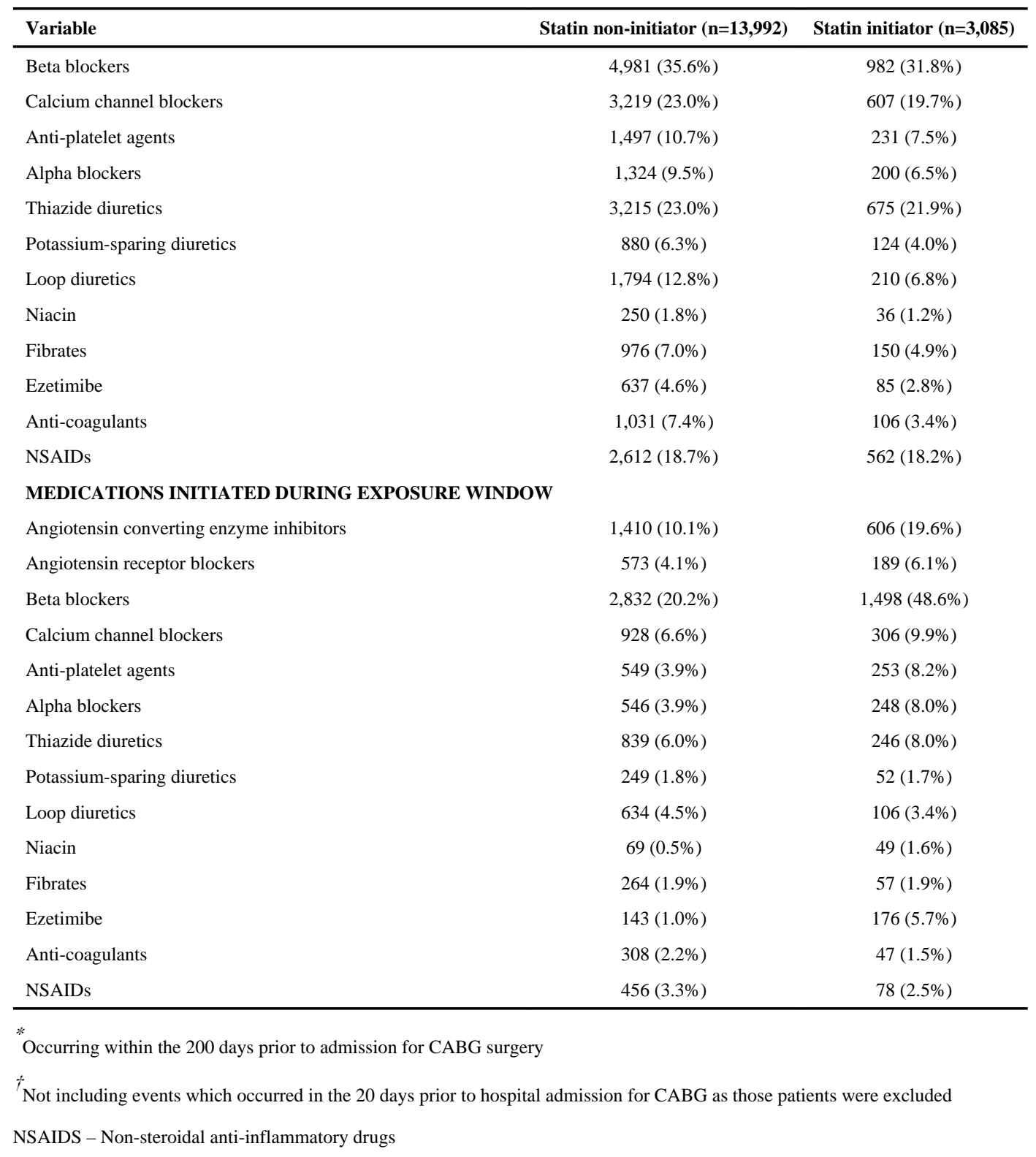


